\title{
An Approach to Tracking Problem for LINEAR CONTROL SYSTEM VIA INVARIANT ELLIPSOIDS METHOD
}

\author{
Mikhail Khlebnikov \\ Laboratory of Adaptive and Robust Systems, \\ V. A. Trapeznikov Institute of Control Sciences of Russian Academy of \\ Sciences, Moscow, Russia
}

\begin{abstract}
In this paper, a simple yet universal approach to the tracking problem for linear control systems via the linear static combined feedback is proposed. The approach is based on the invariant ellipsoid concept and LMI technique, where the optimal control design reduced to finding the minimal invariant ellipsoid for the closed-loop system. With such an ideology, the control design problem directly reduces to a semidefinite programming and one-dimensional minimization. Another attractive property of the proposed approach is that it is equally applicable to discrete-time control systems. The efficacy of the technique is illustrated via a benchmark problem.
\end{abstract}

\section{KEYWORDS}

Linear Control Systems, Tracking Problem, Invariant Ellipsoids, LMIs

\section{INTRODUCTION}

Tracking problem is known as one of the main problem of modern control theory, it is investigated in a lot of papers; see, for instance, $[1,2,3]$. In the most common statement, the tracking problem (so-called, output tracking) supposes the construction the system input or the control law for the dynamical system, such that the system output following the desired function. For the linear system we should to mention the classical monograph [4], see also [5].

There are various tracking problem statements and corresponding approaches. We note classical linear optimal control with linear tracking control [1]; non-linear tracking problem [2]; natural tracking control [3]; Approximating Sequence Riccati Equations (ASRE), etc. Within the context of the $l_{1}$-theory, the problem of finding an accurate estimate of robust system tracking performance, given the information on the nominal model and upper bounds on system uncertainties and disturbances, is solved.

In this paper we propose an approach to one of the statements of the tracking problem. This approach is based on invariant ellipsoids method $[6,7]$. It is easily implemented technically, deals with constraints on the control magnitude, and has a huge potential for possible extensions. The

Dhinaharan Nagamalai et al. (Eds) : SAI, NCO, SOFT, ICAITA, CDKP, CMC, SIGNAL - 2019 pp. 187-198, 2019. (C) CS \& IT-CSCP 2019

DOI: $10.5121 /$ csit.2019.90714 
most close article to the recent paper is [8], which is also devoted to linear tracking problem. However in [8], the input signal is supposed to satisfy a certain differential equation which includes unknown-but-bounded disturbance. In the present paper we only suppose that the input signal and its derivative is bounded; in this way we can consider much more wide class of input signals.

As a technical tool we adopt the powerful LMI technique $[9,10]$ which allows to reduce the stated problem to finding the minimal invariant ellipsoid for the system state. From the computational point of view, the stated problem is reduced to semi-definite programming and one-dimensional optimization. Such problems can be effectively solved computationally using software including (but not limited to) freeware Matlab-based packages SDPT3 [11, 12], YALMIP [13] and cvx [14, 15].

\section{Statement of the Problem}

Let us consider the linear continuous-time control system

$$
\dot{x}=A x+B u+D f, \quad x(0)=x_{0},
$$

where $A \in \mathbb{R}^{n \times n}, B \in \mathbb{R}^{n \times p}, D \in \mathbb{R}^{n \times n}$ are given constant matrices; $x(t) \in \mathbb{R}^{n}$ is the system state, $u(t) \in \mathbb{R}^{p}$ is the control input, and $f(t) \in \mathbb{R}^{n}$ is the input signal such that

$$
\left\|\left(\begin{array}{c}
f \\
\dot{f}
\end{array}\right)\right\| \leq \gamma \quad \text { for any } t \geq 0 \text {. }
$$

It is worth mentioning that there are no other constraints on the input signal $f(t)$. The pair $(A, D)$ is supposed to be controllable.

Here and further, $\mathrm{P} \cdot \mathrm{P}$ is the Euclidean vector norm; ${ }^{\mathrm{T}}$ is the transpose operator; $\operatorname{tr}$ is the matrix trace; $I$ is the identity matrix of the appropriate dimension, and all matrix inequalities are understood in the sense of matrix sign-definiteness.

Our goal is to design the linear static feedback which a) stabilizes the linear system (1)-(2), i.e. makes the system matrix Hurwitz (which guarantees the boundedness of the system trajectories), and b) minimizes the error

$$
e=x-f \in \mathbb{R}^{n} ;
$$

minimization criteria will be discussed later.

Via straightforward differentiation of (3) we obtain the equation

$$
\dot{e}=A e+B u+(A+D) f-\dot{f} .
$$


Introducing the vector

$$
w=\left(\begin{array}{c}
f \\
\dot{f}
\end{array}\right) \in \mathbb{R}^{2 n},
$$

we arrive at

$$
\dot{e}=A e+B u+D_{0} w,
$$

where

$$
\|w(t)\| \leq \gamma \quad \text { for any } t \geq 0
$$

and

$$
D_{0}=\left(\begin{array}{ll}
A+D & -I
\end{array}\right) \text {. }
$$

Let us assume that the values of $e(\tau)$ and $w(\tau)$ are known at any time instant $\tau$, therefore they can be used for feedback design. Namely, we will design the combined feedback (see [16])

$$
u=K_{1} e+K_{2} w,
$$

Where $K_{1} \in \mathbb{R}^{p \times n}, K_{2} \in \mathbb{R}^{p \times 2 n}$.

Due to the joint boundedness of $f(\tau)$ and $\dot{f}(\tau)$ it is natural to treat the input signal $w$ in (4) as an external disturbance.

\section{TECHNiCAL RESUltS}

We use some known results concerned with the linear matrix inequalities $[9,10]$ and invariant ellipsoid technique [6]. The invariant sets are widely used in various guaranteed estimations, filtering and minimax control problems in presence of disturbances. We note the following essential works in the mentioned fields: F. Schweppe [17], D. Bertsekas and I. Rhodes [18], and F. Chernousko [19].

Consider the linear dynamic system

$$
\dot{x}=A x+D w,
$$

where $A \in \mathbb{R}^{n \times n}, D \in \mathbb{R}^{n \times m}$ are fixed known matrices, $x(t) \in \mathbb{R}^{n}$ is the system state, $w(t) \in \mathbb{R}^{m}$ is the external disturbance bounded at any time instance:

$$
\|w(t)\| \leqslant 1 \text { for any } t \geq 0 \text {. }
$$

Any other constraints are not imposed on the disturbance $w(t)$; for instance, it is not supposed to 
be random or harmonic. It is worth mentioning that a more general constraint

$$
\|w(t)\| \leq \gamma \quad \text { for any } t \geq 0
$$

can be reduced to the considered cased by corresponding scaling of the matrix $D$.

Let us suppose that system (6) is stable ( $A$ is the Hurwitz matrix), the pair $(A, D)$ is controllable.

Definition 1. Ellipsoid centred at the origin

$$
\mathcal{E}_{P}=\left\{x \in \mathbb{R}^{n}: \quad x^{\mathrm{T}} P^{-1} x \leq 1\right\}, \quad P \succ 0,
$$

is called invariant for the system (6)-(7), if from $x(0) \in \mathcal{E}_{P}$ follows $x(t) \in \mathcal{E}_{P}$ for any $t \geq 0$ and all admissible disturbances $w(t)$.

In other words, any trajectory of the system that comes from the point lying in the ellipsoid $\mathcal{E}_{P}$ belongs to this ellipsoid at any time instant. The matrix $P$ is called the ellipsoid matrix.

Theorem 1 ([9]). Ellipsoid $\mathcal{E}_{P}$ is invariant for the dynamical system (6)-(7) if and only if the ellipsoid matrix $P \succ 0$ satisfies the linear matrix inequality

$$
A P+P A^{\mathrm{T}}+\alpha P+\frac{1}{\alpha} D D^{\mathrm{T}} \preceq 0
$$

for a certain $\alpha>0$.

Invariant ellipsoid can be treated as effective tool for the estimation of state of the dynamical system subjected to bounded disturbances.

Disturbance influence can be characterised by minimal invariant ellipsoid. Among the various minimality criteria we will use the trace criterion $\operatorname{tr} P$ which corresponds to the sum of squared semi-axes of the ellipsoid matrix $P$.

\section{Main Result}

Let us turn back to the minimization of the error

$$
e=x-f
$$

for system (1). We will seek the minimal invariant ellipsoid for system (4) embraced with feedback (5). In this way the system takes the following closed-loop form

$$
\dot{e}=A_{c} e+D_{c} w,
$$

where 


$$
A_{c}=A+B K_{1}, \quad D_{c}=D_{0}+B K_{2} .
$$

The next theorem presents the main result of the paper.

Theorem 2. Let $P, Y, K_{2}$ be the solution of the minimization problem

$$
\min \operatorname{tr} P
$$

subject to the constraints

$$
\left(\begin{array}{cc}
A P+P A^{\mathrm{T}}+\alpha P+B Y+Y^{\mathrm{T}} B^{\mathrm{T}} & * \\
\gamma\left(D_{0}+B K_{2}\right)^{\mathrm{T}} & -\alpha I
\end{array}\right) \preceq 0,
$$

with respect to the matrix variables, , $P=P^{\mathrm{T}} \in \mathbb{R}^{n \times n}, Y \in \mathbb{R}^{p \times n}, K_{2} \in \mathbb{R}^{p \times 2 n}$ and the scalar parameter $\alpha$.

Then the combined controller (5) with matrix

$$
\left(\begin{array}{ll}
Y P^{-1} & K_{2}
\end{array}\right)
$$

stabilizes system (4), and $P$ is an invariant ellipsoid matrix for the closed-loop system with zero initial condition.

Applying Theorem 1 for the system (8) and minimizing invariant ellipsoid with matrix $P$, we arrive at the following minimization problem

$$
\min \operatorname{tr} P
$$

subject to the constraints

$$
\left(A+B K_{1}\right) P+P\left(A+B K_{1}\right)^{\mathrm{T}}+\alpha P+\frac{1}{\alpha} \gamma^{2}\left(D_{0}+B K_{2}\right)\left(D_{0}+B K_{2}\right)^{\mathrm{T}} \preceq 0
$$

and

$$
P \succ 0 \text {. }
$$

Using Schur lemma [20], the first constraint can be reformulated as

$$
\left(\begin{array}{cc}
\left(A+B K_{1}\right) P & \\
+P\left(A+B K_{1}\right)^{\mathrm{T}}+\alpha P & \gamma\left(D_{0}+B K_{2}\right) \\
\gamma\left(D_{0}+B K_{2}\right)^{\mathrm{T}} & -\alpha I
\end{array}\right) \preceq 0 .
$$

Let us introduce the auxiliary variable 


$$
Y=K_{1} P
$$

to eliminate $K_{1}$.

Due to $P \succ 0$, the matrix $K_{1}$ can be restored in the unique way:

$$
K_{1}=Y P^{-1}
$$

The proof is complete.

We make several comments.

1. There are both strict and nonstrict inequalities in optimization problem in the theorem above. Such specific is common for used technique and it described in details in [10].

2. It is natural to require that the control is bounded, for instance, by imposing a straightforward constraint like

$$
\|u(t)\| \leq \mu,
$$

see [21]. In the present paper we introduce the constraint applied to the first control component (5):

$$
\left\|K_{1} e\right\| \leq \mu, \quad \mu>0
$$

As the parameter $K_{2}$ is the variable of the optimization problem (9)-(10), we can impose the constraint directly (if needed).

As shown in [9], the condition (11) is guaranteed by the fulfillment of the LMI

$$
\left(\begin{array}{cc}
P & Y^{T} \\
Y & \mu^{2} I
\end{array}\right) \succeq 0 .
$$

This constraint is to be added to the constraints of the theorem.

3. For any fixed value of the parameter $\alpha$, the minimization problem (9)-(10) is a semi-definite programm. It is possible to identify the range for $\alpha$.

Namely, let us consider the following optimization problem

$$
\min \lambda
$$


subject to the constraints

$$
\begin{aligned}
& \left(\begin{array}{cc}
A P+P A^{\mathrm{T}}+\alpha P+B Y+Y^{\mathrm{T}} B^{\mathrm{T}} & \gamma\left(D_{0}+B K_{2}\right) \\
\gamma\left(D_{0}+B K_{2}\right)^{\mathrm{T}} & -\alpha I
\end{array}\right) \preceq 0, \\
& \left(\begin{array}{cc}
P & Y^{T} \\
Y & \lambda I
\end{array}\right) \succeq 0, \quad P \succ 0,
\end{aligned}
$$

with respect to the matrix variables $P=P^{\mathrm{T}} \in \mathbb{R}^{n \times n}, Y \in \mathbb{R}^{p \times n}, K_{2} \in \mathbb{R}^{p \times 2 n}$, the scalar variable $\lambda$ and the scalar parameter $\alpha>0$.

This problem is a semi-definite programm and it is feasible for any positive $\alpha$, thus [10],

$$
\mu_{\min }(\alpha)=\sqrt{\lambda_{\min }(\alpha)}
$$

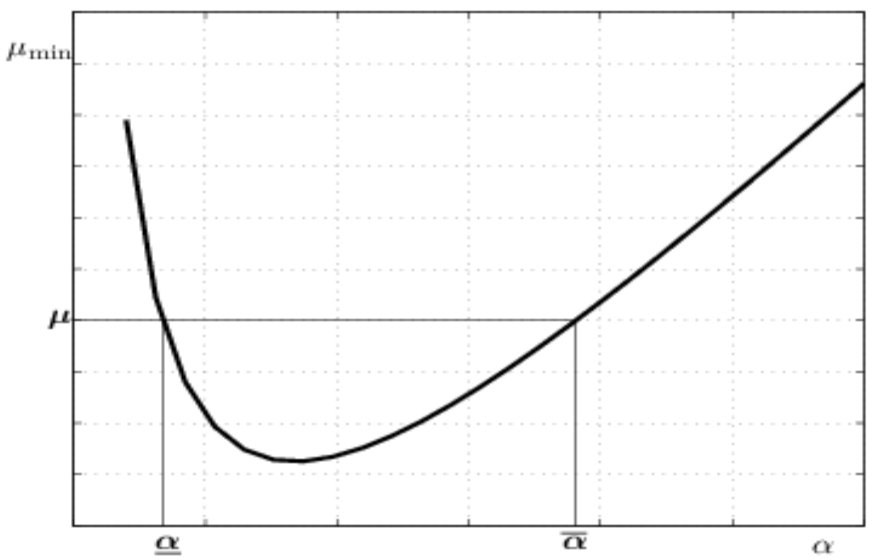

Figure 1. Plot of $\mu_{\min }(\alpha)$.

Fig. 1 depicts the plot of the function $\mu_{\text {min }}(\alpha)$ for a sample system.

The projection of the cross-section of the epigraph the function $\mu_{\text {min }}(\alpha)$ at level $\mu$ on the horizontal axis gives us the corresponding range $[\underline{\alpha}, \bar{\alpha}]$ for the parameter $\alpha$.

4. The conditions stated in the theorem are sufficient only, therefore the obtained solution is suboptimal. 


\section{EXAMPLE}

We demonstrate the efficacy of the proposed approach via a benchmark problem. The numerical values were taken from AC11 task of the COMPleib library [22]:

$$
\begin{gathered}
A=\left(\begin{array}{ccccc}
-1.341 & 0.9933 & 0 & -0.1689 & -0.2518 \\
43.223 & -0.8693 & 0 & -17.251 & -1.5766 \\
1.341 & 0.0067 & 0 & 0.1689 & 0.2518 \\
0 & 0 & 0 & -20 & 0 \\
0 & 0 & 0 & 0 & -20
\end{array}\right), \\
D=\left(\begin{array}{ccc}
0 & 0 \\
0 & 0 \\
0 & 0 \\
20 & 0 \\
0 & 20
\end{array}\right), \\
\left.0 \begin{array}{ccccc}
0 & 0 & 1 & 0 & 0 \\
47.76 & -0.268 & 0 & -4.56 & 4.45 \\
0 & 0 & 0 & 1 & 0 \\
0 & 0 & 0 & 0 & 1 \\
0 & 0 & 0 & 0 & 0
\end{array}\right) .
\end{gathered}
$$

The solution of the optimization problem from Theorem 2 for $\gamma=1$ and $\mu=5$ is obtained with

$$
\alpha=6.6
$$

we arrive at the matrix

$$
P=\left(\begin{array}{ccccc}
1.8083 & -2.1786 & -0.8381 & 5.0692 & 3.5415 \\
* & 49.171 & -0.6509 & 40.1701 & -2.3267 \\
* & * & 0.6105 & -0.8683 & -4.2103 \\
* & * & * & 120.707 & -35.5206 \\
* & * & * & * & 47.381
\end{array}\right)
$$

of the invariant ellipsoid, and the gain matrices 
and

$$
K_{1}^{\mathrm{T}}=\left(\begin{array}{cc}
1245.1 & -1685.9 \\
147 & -198.6 \\
1935 & -2636.1 \\
-79.3 & 106.9 \\
27.1 & -37.5
\end{array}\right)
$$

$$
K_{2}^{\mathrm{T}}=\left(\begin{array}{cc}
7.7995 & -3.261 \\
0.5486 & -0.3446 \\
0.6452 & -0.362 \\
-0.1652 & -1.0632 \\
0.2047 & 0.6886 \\
-0.6452 & 0.362 \\
-0.0851 & 0.0212 \\
-0.685 & 1.357 \\
0.05 & 0 \\
0 & 0.05
\end{array}\right) .
$$

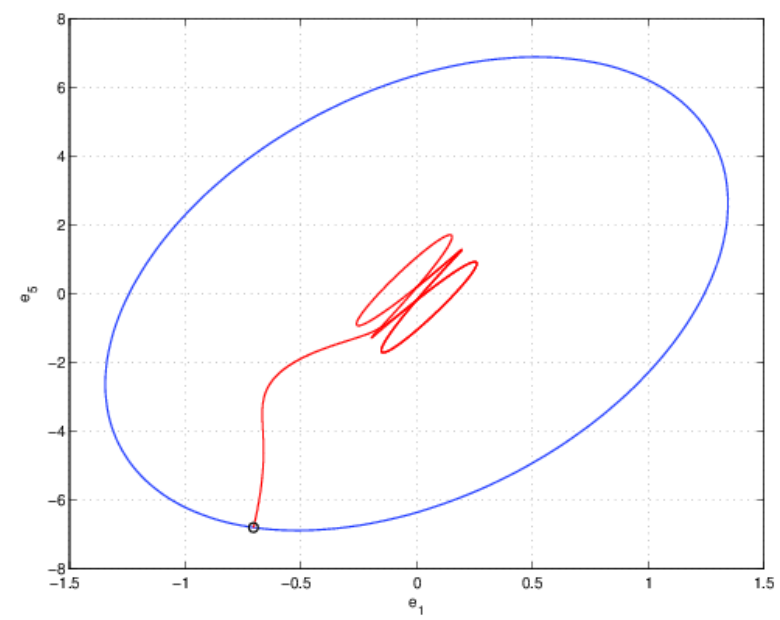

Figure 2. Invariant ellipsoid and projections of the phase trajectory.

Fig. 2 depicts the projection of the invariant ellipsoid for the closed-loop system (8) on the plane $\left(e_{1}, e_{5}\right)$ and the corresponding trajectory projection.

Fig. 3 depicts the dynamics of the control components for the input signal 


$$
f(t)=\frac{1}{\sqrt{2}}\left(\begin{array}{c}
0 \\
\sin (t / 3) \\
0 \\
\cos t \\
0
\end{array}\right) .
$$

The plot of the component $u_{1}(t)$ is shown in blue, and $u_{2}(t)$ in red.

Fig. 4 depicts the dynamics of the norm $\|u(t)\|$.

We used MATLAB-based packages SDPT3 and YALMIP for numerical simulations.

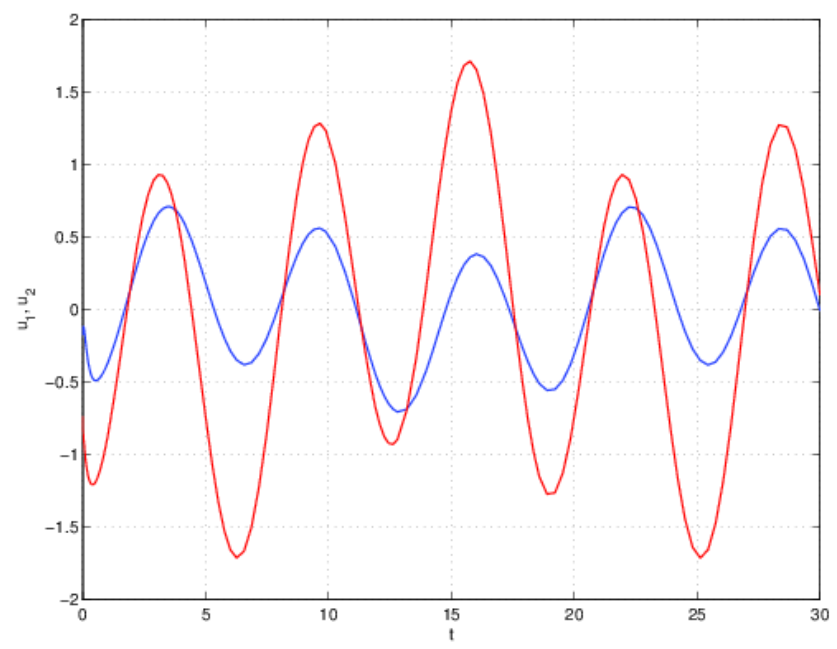

Figure 3. Dynamics of control components.

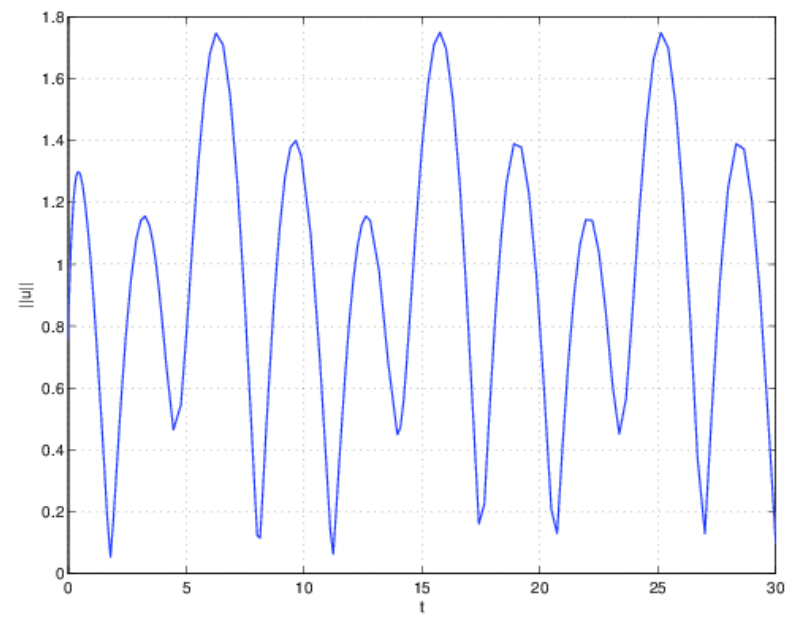

Figure 4. Plot of $\|u(t)\|$. 


\section{Conclusions}

A simple but universal approach for one statement of the linear tracking problem is supposed. The approach is based on the invariant ellipsoid technique and linear matrix inequalities apparatus. The control design of linear static combined feedback was reduced to semi-definite programming and one-dimensional optimization. The approach efficacy is demonstrated via numerical simulation.

The future plans are to adopt the approach to discrete-time systems, to robust statements (with structured uncertainties in system matrices), and to systems subjected to bounded exogenous disturbances.

\section{ACKNOWLEDGEMENTS}

This work was partially supported by the Russian Foundation for Basic Research, project no. 1808-00140 and by the Russian Science Foundation, project no. 16-11-10015.

The author is grateful to Kirill Zheleznov for performing the numerical simulations.

\section{REFERENCES}

[1] M. Athans \& P. L. Falb (1966), Optimal Control: An Introduction to the Theory and Its Applications, McGraw-Hill, New York.

[2] T. Cimen \& S. Banks (2004), Nonlinear optimal tracking control with application to super-tankers for autopilot design, Automatica, Vol. 40, No. 11, pp. 1845-1863.

[3] L. T. Grujic \& W. P. Mounfield (1992), Natural tracking PID process control for exponential tracking, AIChE Journal, Vol. 38, No. 4, pp. 555-562.

[4] L. T. Gruyitch (2013), Tracking Control of Linear Systems, CRC Press, Boka Raton.

[5] Y. Choi \& W. K. Chung (2004), PID Trajectory Tracking Control for Mechanical Systems. SpringerVerlag, Berlin.

[6] M. V. Khlebnikov \& B. T. Polyak \& V. M. Kuntsevich (2011), Optimization of linear systems subject to bounded exogenous disturbances: The invariant ellipsoid technique, Automation and Remote Control, Vol. 72, No. 11, pp. 2227-2275.

[7] S. A. Nazin \& B. T. Polyak \& M. V. Topunov (2007), Rejection of bounded exogenous disturbances by the method of invariant ellipsoids, Automation and Remote Control, Vol. 68, No. 3, pp. 467-486.

[8] K. O. Zheleznov \& M. V. Khlebnikov (2013), Applying invariant ellipsoid technique for linear tracking problem, Proceedings of Moscow Institute of Physics and Technology (in Russian), Vol. 5, No. 4, pp. 115-121.

[9] S. Boyd \& L. El Ghaoui \& E. Feron \& V. Balakrishnan (1994), Linear Matrix Inequalities in System and Control Theory, SIAM, Philadelphia. 
[10] B. T. Polyak \& M. V. Khlebnikov \& P. S. Shcherbakov (2014), Control of Linear Systems Subjected to Exogenous Distrubances: An LMI Approach, LENAND, Moscow.

[11] K. C. Toh \& M. J. Todd \& R. H. Tütüncü (1999), SDPT3 - a Matlab software package for semidefinite programming, Optimization Methods and Software, Vol. 11, No. 1-4, pp. 545-581.

[12] R. H. Tütüncü \& K. C. Toh \& M. J. Todd (2003), Solving semidefinite-quadratic-linear programs using SDPT3, Mathematical Programming Series, Vol. 85, No. 2, pp. 189-217.

[13] J. Löfberg (2004), YALMIP: A toolbox for modeling and optimization in Matlab, Proc. of the 13th IEEE International Symposium on Computer Aided Control System Design (CACSD 2004).

[14] CVX Research, Inc. (2012), CVX: Matlab software for disciplined convex programming, version 2.0, URL http://cvxr.com/cvx.

[15] M. Grant \& S. Boyd (2008), Graph implementations for nonsmooth convex programs, In V. Blondel, S. Boyd, and H. Kimura, editors, Recent Advances in Learning and Control, Lecture Notes in Control and Information Sciences, pp. 95-110, Springer-Verlag Limited, URL http://stanford.edu/ boyd/graph_dcp.html.

[16] M. V. Khlebnikov (2016), Control of linear systems subjected to exogenous disturbances: Combined feedback, Proc. of the 12th IFAC International Workshop on Adaptation and Learning in Control and Signal Processing (ALCOSP 2016), pp. 111-116.

[17] F. C. Schweppe (1973), Uncertain Dynamic Systems, Prentice Hall, NJ.

[18] D. P. Bertsekas \& I. B. Rhodes (1971), Recursive state estimation for a set-membership description of uncertainty, IEEE Transactions on Automatic Control, Vol. 16, pp. 117-128.

[19] F. L. Chernousko (1988), State Estimation for Dynamic Systems, Nauka, Moscow.

[20] R. Horn \& C. Johnson (1990), Matrix Analysis, Cambridge University Press.

[21] M. V. Khlebnikov \& P. S. Shcherbakov (2014), Optimal feedback design under bounded control, Automation and Remote Control, Vol. 75, No. 2, pp. 320-322.

[22] F. Leibfritz (2004), COMPleib: COnstraint Matrix-optimization Problem library - a collection of test examples for nonlinear semidefinite programs, control system design and related problems, URL http://www.complib.de/.

\section{AUTHOR}

Mikhail Khlebnikov was born in 1973 in Moscow, Russia. He is Doctor of Science in Physics and Mathematics (Highest scientifical degree in Russia) from Institute of Control Sciences RAS (2010). At the present time he is the Head of the Ya. Z. Tsypkin Laboratory of Adaptive and Robust Systems at V. A. Trapeznikov Institute of Control Sciences of Russian Academy of Sciences (ICS RAS).

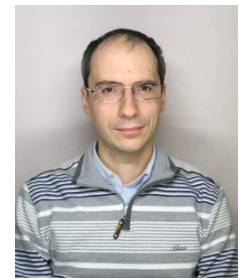

